\title{
McMaster Brealkthrough May One Day Decrease Patient Reliance on Blood Donors
}

Sean McFadden (McMaster University)

News Reporter - HSI 2OIO-2OII

A groundbreaking discovery at McMaster University shows great promise in eliminating the reliance upon blood and marrow transplants by cancer patients. Dr. Mick Bhatia and his research team recently discovered that a specific protein cocktail has the potential to transform adult skin cells directly into platelets and red blood cells. Dr. Bhatia is a Canadian leader in stem cell biology and the director of McMaster's Stem Cell and Cancer Research Institute (MSCCRI). Dr. Bhatia's finding has made him a pioneer in the stem cell field, allowing scientists to transform human skin directly into functional blood cells. This discovery will have profound ramifications for the field of stem cell biology. It provides hope for cancer patients, especially those suffering from leukemia, who rely heavily upon blood bank donations.

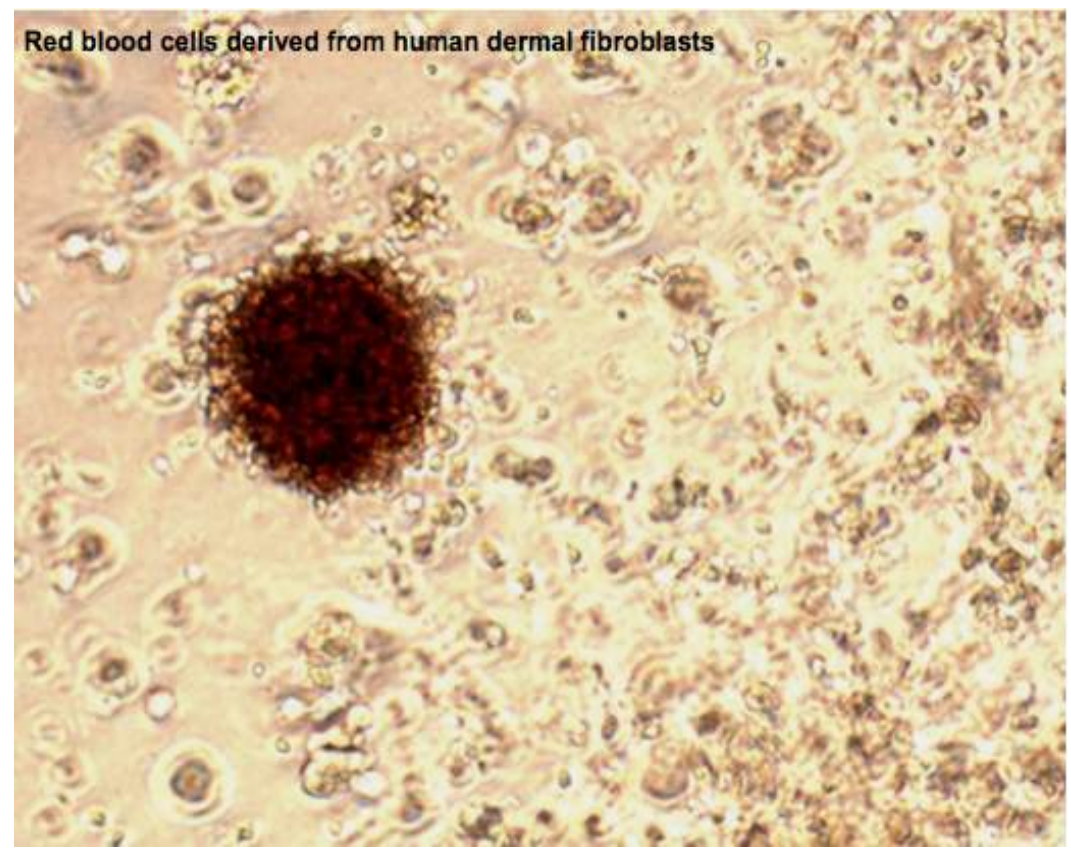

When asked what previous research was most influential to his recent discovery, Dr. Bhatia identified two studies. The first demonstrated the potential of stem cell research, when lan Wilmut cloned the first mammal, a sheep named 'Dolly.' "This told us a very important thing: that cells can reprogram. It showed that DNA is very plastic and can be manipulated." The next important finding came from Shinya Yamanaka, who showed that fully mature cells from a mouse or human could be reverted to a pluripotent state, meaning that the cell has the potential to turn into many different tissue types. "This finding showed that scientists could create in a lab, at a cellular level, the events that occur during development, inducing cells to become specific tissue types." These two studies paved the way for future breakthroughs in stem cell research and provided the means to study cancer using stem cells.

The process of direct conversion to mature blood cells

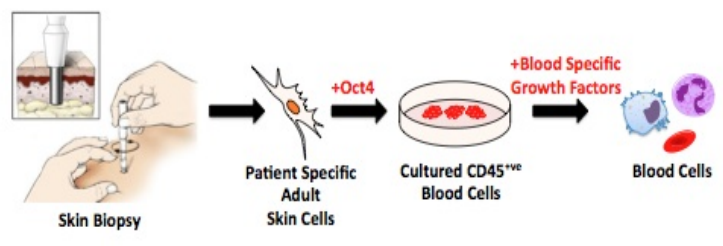

The insights that can be gained from studying stem cells and their effects on cancer treatment are twofold. Firstly, it is important to note that the most significant thing that a cancer cell does is proliferate. This is also an inherent trait shared with undifferentiated stem cells. Stem cells in their normal condition need to stay in the self-renewal process while also staying undifferentiated; this is an important feature shared by cancer and stem cells, and is something that researchers at the MSCCRI are currently trying to exploit. "When you think of the analogy of a car with an accelerator and a brake, cancer cells are not able to hit the brake, whereas stem cells can. It is the task of scientists to find out what this brake is to stop the process of cell proliferation." Slowing or stopping the replication of cancer cells would allow for more specialized chemotherapy and treatment options for those battling cancers. 
The second insight gained from studying stem cells, useful for improving cancer treatment techniques, is deciphering what factors are necessary to commit developing cells to a specific tissue type. This issue is addressed in Dr. Bhatia's most recent publication. "What our group set out to accomplish was to look empirically through many different transcription factors to identify specific genetic and epigenetic changes which would allow us to differentiate skin cells directly into blood, without reverting first to a pluripotent state." Differentiating the skin directly into blood is important for a number of reasons. One reason is that by doing so, this method removes the risks associated with the use of pluripotent cells, one of which is the formation of dangerous tumors. Dr. Bhatia's method is also unique because the use of adult tissues does not carry the same ethical stigma as embryonic stem cells.

The method for inducing skin cells to differentiate into other functional cell types provides scientists the opportunity to begin creating other important tissues. In fact, Dr. Bhatia's lab is already pursuing the task of transforming adult tissue into neural cells. Such an achievement could help the scientific community understand and create more focused treatments for neurological illnesses such as Huntingon's and Parkinson's disease. Dr. Bhatia's laboratory is also investigating the potential conversion of skin cells directly into white blood cells of the lymphocyte lineage. These are the $B$ and $T$ cells which provide the body with its immunity against infection. A readily available source of these white blood cells could one day help to treat patients who are immunocompromised, particularly those undergoing chemotherapy or suffering from AIDS.

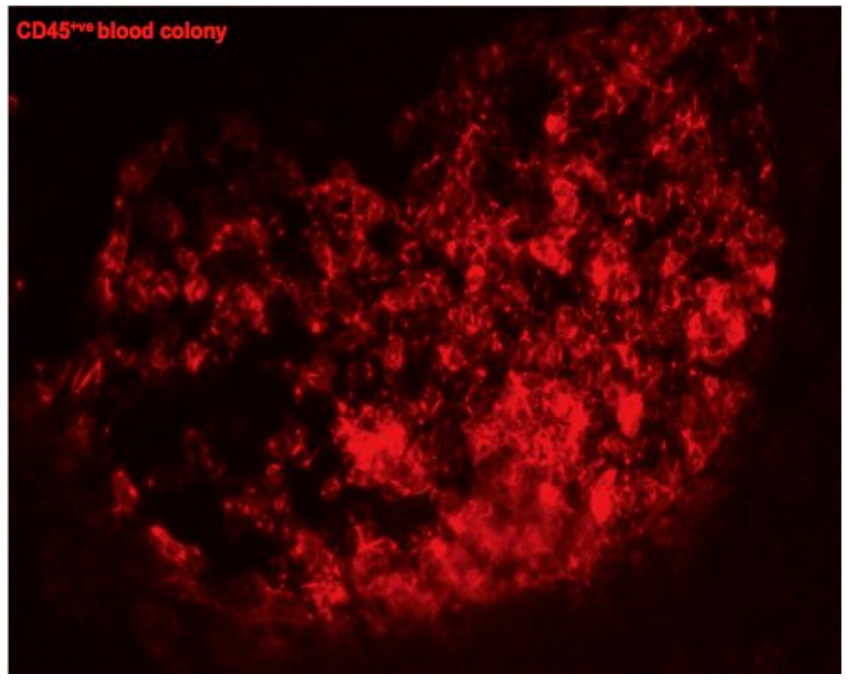

This recent discovery from the Bhatia lab emphasizes the importance of stem cell research in contributing to the current state of knowledge on illnesses such as cancer, as well as the cells' ultimate potential as an effective treatment option.

Images provided by Dr. Mick

Bhatia, director and senior

scientist at McMaster's Stem

Cell and Cancer Research

Institute.

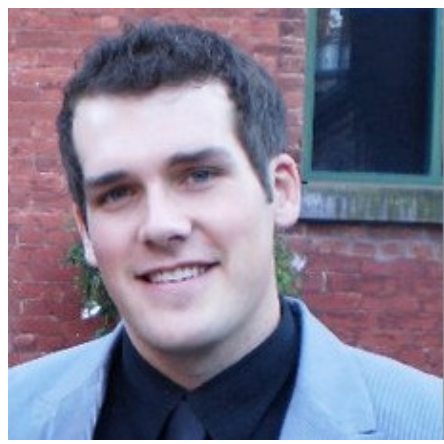

News Reporter Profile

Sean McFadden is currently pursuing a MSc degree in Physiology from the University of Toronto. His project is investigating the mechanisms through which hormones regulate neuropeptide production and secretion in the hypothalamus using immortalized hypothalamic cell lines. His research interests include diabetes and obesity disease onset and progression, as well as reproductive disorders associated with these pathologies. 